grammes are meeting this target. The number failing to do so is little more than expected from chance variation, and many regions now exceed it, which has led to adoption of a new "achievable" standard of $6 / 1000$. However, only six of the 17 regions and countries and $32 \%$ of individual programmes reached the target of at least 1.5 small breast cancers (diameter $\leqslant 10 \mathrm{~mm}$ ) per 1000 screened. This criterion is used both as an indicator of the quality of mammography and as an indicator of the long term change in prognosis which, it is hoped, will be achieved by screening. More experience is needed to assess whether the detection rate of 3.4 breast cancers per 1000 at the three yearly repeat screen is sufficient to keep down the rate of interval cancers to $25 \%$ or less of expected three yearly incidence. ${ }^{6}$ All programmes are now setting up mechanisms for the routine reporting of interval cancers and cancers in non-responding eligible women from regional cancer registries and in some cases pathology laboratories. These will enable longer term predictions of whether the national screening programme is on course to meet its Health of the Nation target of a $25 \%$ reduction of breast cancer mortality in eligible women by the year $2000 .^{7}$

We are grateful to the administrative staff in the numerous screening offices who ran the original tables for their patience in responding to our numerous queries; to the radiologists who provided data for "the big 18"; to the regional and national coordinators who helped us to chase up missing data; to Professor Martin Vessey, chairman of the Department of Health's Advisory Committee on Breast Cancer Screening, and Mrs Julietta Patnick, National Coordinator of NHS breast screening programme for their encouragement; and to Mr Richard Yates of Department of Health's Statistics Division. The Cancer Screening Evaluation Unit at the Institute of Cancer Research is supported by the Department of Health Research and Development Section.

1 Forrest APM. Breast cancer screening: report to the health ministers of England, Wales, Scotland, and Northern Ireland. London: HMSO, 1986.

2 Department of Health. Guidelines on the establishment of a quality assurance system for the radiological aspects of mammography used in breast screening. Sheffield: NHSBSP Publications, Trent Regional Health Authority, 1988

3 UK Trial of Early Detection of Breast Cancer Group. Breast cancer mortality after ten years in the UK trial of early detection of breast cancer. The Breast 1993;2:13-20.

4 Report of the UKCCCRNHSBSP workshop on acceptability research. Sheffield: NHSBSP (in press)

5 Frisell J, Eklund G, Hellstrom L, Lidbrink E, Rutqvist L-E, Somell A Randomized study of mammography - preliminary report of mortality in the Stockholm trial. Breast Cancer Research and Treatment 1991;18:49-56.

6 Tabar L, Fagerberg G, Day NE, Holmberg L. What is the optimum interval between mammographic screening examinations? Br $f$ Cancer 1987;55: between

7 Secretary of State for Health. The Health of the Nation. A strategy for health in England. London: HMSO, 1992

(Accepted 25 fune 1993)
Academic Department of Public Health, St Mary's Hospital Medical School, London W2

Helen Ward, lecturer

Sophie Day, senior research

fellow

Jane Mezzone, research assistant

Lucinda Dunlop, research registrar

Catrina Donegan, research nurse

Sara Farrar, research assistant Luke Whitaker, research fellow

David L Miller, head of

department

\section{Department of} Genitourinary Medicine, St Mary's Hospital Medical School

John R W Harris, consultant

Correspondence to: DrWard.

\title{
Prostitution and risk of HIV: female prostitutes in London
}

\section{Helen Ward, Sophie Day, Jane Mezzone, Lucinda Dunlop, Catrina Donegan, Sara Farrar, Luke Whitaker, John R W Harris, David L Miller}

Abstract

Objective-To measure the prevalence of HIV and to describe established risk factors in female prostitutes.

Design-A cross sectional survey.

Setting-A genitourinary medicine clinic, streets, and magistrates' courts in London.

Subjects-280 female prostitutes recruited between April 1989 and August 1991.

Main outcome measures-Infection with HIV-1, reported risk behaviours, and prevalence of sexually transmitted infections.

Results-228 of the women had HIV tests, and two $(0.9 \%(95 \%$ confidence interval $0 \%$ to $2 \cdot 1 \%))$ were infected with HIV-1. Reported use of condoms was high for commercial clients and low for non-paying partners: $98 \%(251 / 255)$ of women used condoms with all clients and $12 \%(25 / 207)$ with non-paying partners for vaginal intercourse. Twenty two women were current or past injecting drug users. Of the 193 women examined for sexually transmitted infections, 27 had an acute infection (gonorrhoea, chlamydia, trichomonas, or primary genital herpes) at the time of interview. Infection was associated with younger age and increasing numbers of non-paying sexual partners, but not with duration of prostitution, numbers of clients, or reports of condom failures. When age and numbers of non-paying partners were analysed by logistic regression they remained significantly associated with sexually transmitted infections.

Conclusions-A large and diverse sample of prostitutes had a low prevalence of infection with HIV and high levels of use of condoms in commercial sex. There was a significant risk of other sexually transmitted infections associated with prostitutes' non-commercial sexual relationships, in which unprotected sex is common. Interventions to reduce the risk of sexually transmitted infections in prosti- tutes should address both commercial and noncommercial sexual partnerships.

\section{Introduction}

It is unclear whether prostitute women in Europe are at risk of heterosexually acquired HIV independently of use of injected drugs. The risk of HIV infection has been highest in those who have a history of use of injected drugs which is associated with a history of street prostitution. ${ }^{1-3}$

A prevalence of infection with HIV-1 of $1.6 \%$ was reported in 188 female prostitutes studied during $1986-8$ in a London genitourinary medicine clinic. The work reported here was designed to show whether this low prevalence was related to the stage of the epidemic, sampling bias, or effective risk avoidance through the use of condoms.

\section{Subjects and methods}

A representative sample of prostitutes is difficult to define, but we had previously described the major sectors of prostitution in London ${ }^{5}$ and we developed methods of recruitment to ensure that our sample included women working in all these sectors. Between April 1989 and August 1991 prostitutes were recruited by referral from health workers in the genitourinary medicine clinic at St Mary's Hospital, referral from friends and colleagues of prostitutes, fieldwork (visiting streets, magistrates' courts, flats, agencies, and saunas), and telephone. We aimed for a sample size of at least 200 women to detect a prevalence as low as $2 \%$ with a $95 \%$ confidence interval that did not include zero.

A questionnaire asking for information on medical, social, and prostitution history was administered by trained interviewers. All participants were offered a HIV test, although acceptance was not a condition for 
participation in the study. Those who did not want a test were invited to provide a saliva or blood sample for anonymous testing with results available only to the principal investigator at the time of analysis. HIV tests were performed on serum samples at the Department of Virology, St Mary's Hospital, and on saliva samples at the Central Public Health Laboratory. Both laboratories used enzyme linked immunosorbent assay (ELISA) followed by immunoblot confirmation. ${ }^{6}$ Participants were also offered genital screening for sexually transmitted infections. Tests were carried out for Neisseria gonorrhoeae (gram stain and culture), Chlamydia trachomatis (direct immunofluorescence), Trichomonas vaginalis (direct microscopy), and herpes simplex virus (culture).

Confidentiality was maintained by using unique study numbers, allowing only the principal investigatdata were analysed with SAS: categorical variables were compared by $\chi^{2}$ tests; continuous variables (age parametric tests (Mann-Whitney $U$ test) as they were not normally distributed; and multivariate analysis was performed by unconditional logistic regression.

\section{Results}

Of the 280 women who were recruited to the study, 193 were interviewed in the clinic at St Mary's and 87 were recruited during fieldwork-36 on the streets, 35 in magistrates' courts, 10 through a drop in centre, and six in an agency or flat. The participants were aged 1651 (median 25); they had been working as prostitutes for between one month and 34 years (median four years); 228 had been born in the United Kingdom; and $221(80 \%)$ were white, $25(9 \%)$ were Afro-Caribbean, and $29(11 \%)$ were Asian, Middle Eastern, or other (no data were available for five women). Educational level provided a proxy measure of social class for 277 of the women: $22(8 \%)$ left full time education aged 14 or less, $149(54 \%)$ left aged 15 or $16,85(31 \%)$ left aged 17 or 18 , and $21(8 \%)$ left aged over 18 . The sample included women working in all the major sectors of prostitution identified in London (see table 1): those contacted by fieldwork were primarily working on the streets while those interviewed at the clinic were working in all sectors of prostitution.

Altogether 228 women agreed to HIV testing: two $(0.9 \%$ (95\% confidence interval $0 \%$ to $2 \cdot 1 \%))$ were infected with HIV-1, but both had been diagnosed before the study and both reported a history of use of injected drugs. The 52 women who declined HIV

TABLE II-Proportion (percentage) of prostitutes who always used condoms with commercial clients in previous week and with nonpaying partners in previous month by sexual behaviour

\begin{tabular}{lcc}
\hline Sexual behaviour & $\begin{array}{c}\text { Commercial } \\
\text { clients }\end{array}$ & $\begin{array}{c}\text { Non-paying } \\
\text { partners }\end{array}$ \\
\hline Vaginal intercourse & $251 / 255(98)$ & $25 / 207(12)$ \\
Oral sex & $163 / 196(83)$ & $10 / 176(6)$ \\
Anal intercourse & $4 / 8(50)$ & $8 / 32(25)$ \\
\hline
\end{tabular}

TABLE III-Proportion (percentage) of prostitutes with acute sexually transmitted infection (gonorrhoea, chlamydia, trichomonas, or primary genital herpes) by number of non-paying sexual partners in previous month

No of non-paying

partners

\begin{tabular}{cc}
\hline 0 & $3 / 38(8)$ \\
1 & $17 / 107(16)$ \\
$>1$ & $7 / 25(28)$ \\
\hline$\chi^{2}$ Test for trend $=4 \cdot 414, \mathrm{p}=0.036$.
\end{tabular}

testing were compared with those who accepted. They were similar with respect to their ages, the time they had been prostitutes, the sectors of prostitution they worked in, their history of established risk factors for infection with HIV, and their history of infection with hepatitis B. Women who had more years of education were more likely to agree to HIV testing: tests were accepted by $14(64 \%)$ of those who left education aged 14 or less, $119(80 \%)$ of those who left aged 15 or 16 , and $90(85 \%)$ of those who left aged 17 or over $\left(\chi^{2}\right.$ test for trend, $\left.\mathrm{p}=0.038\right)$.

Of the 52 women who declined testing, 34 reported having had HIV tests previously, all with negative ors to link data from interviews to clinical records. The and duration of prostitution) were analysed by non-

results. The 18 women who had never been tested were compared with the rest, and no significant associations were found with age, history of known risk factors, or with numbers of clients or non-paying partners.

Twenty two of the women had a history of blood transfusion; 22 were current or past injecting drug users; 53 reported use of injected drugs either by themselves or by their sexual partners; and 58 reported having sex with bisexual men and four with men known to be infected with HIV. Women recruited through fieldwork were more likely to report use of injected drugs than those interviewed at the clinic $\left(11 / 87(13 \%) v 11 / 193(6 \%) ; \chi^{2}=3 \cdot 99, \mathrm{p}=0.046\right)$.

The women were asked about their clients and their use of condoms in the previous week. A pilot study had shown that this was the most reliable indicator of average numbers of clients and types of sexual behaviour. The median number of clients was nine (0-198), and table II shows the use of condoms for different types of sex. In addition, 211 women reported having sex with one or more non-paying male partners in the past month, and the use of condoms with these partners is also shown in table II. Of these 211 women, 98 reported that their partners were not currently monogamous, and 24 said that the men's other sexual partners had included prostitutes. Altogether 104 women reported one or more condoms failing (leaking or bursting) in the month before the interview.

The women were asked if they had ever had a sexually transmitted infection diagnosed, and 123 reported past infection with gonorrhoea. The women recruited through fieldwork were less likely to report a history of gonorrhoea than those interviewed in the clinic (35/87 (40\%) $v 88 / 190(46 \%))$, but this difference was not statistically significant $\left(\chi^{2}=0 \cdot 9, p=0.34\right)$. In total, 193 women (mainly those interviewed in the clinic) were examined for sexually transmitted infections on the day of their interview or within a week later, and 27 had one or more current, acute infections: nine had gonorrhoea, 12 chlamydia, seven trichomonas, and four primary genital herpes.

The women with an acute sexually transmitted infection were younger than those without (median ages $22 v 26$; Mann-Whitney $\mathrm{U}$ test, $\mathrm{p}=0.0019)$. Acute sexually transmitted infection was also associated with increasing numbers of non-paying sexual partners in the past month (table III) but not with numbers of clients, condom failures, history of use of injected drugs, or level of education. Women's ages and numbers of non-paying partners were analysed together in a logistic regression model (women's ages were grouped by quartiles). The association with sexually transmitted infection remained significant for both age and numbers of non-paying partners: the adjusted odds ratio for each increase in age group over the baseline age group odds was 0.57 (95\% confidence interval 0.37 to 0.88$)(\mathrm{p}=0.011)$, and the adjusted odds ratio for each additional non-paying partner over the odds for none was $1.92(1.04$ to 3.22$)(p=0.036)$.

\section{Discussion}

Our previous report of a low prevalence of infection with HIV in prostitutes in London was based on a relatively small sample recruited from a genitourinary medicine clinic. ${ }^{4}$ While it remains impossible to define a truly representative sample, we have now studied a larger sample of prostitutes, recruited through diverse methods from all sectors of prostitution, and found an equally low prevalence. The high proportion of the participants who agreed to an HIV test and the few identifiable differences between those tested and those not tested add to the confidence of our estimate and reduces concern about possible bias in the earlier sample. 
This finding contrasts with recent press reports of high levels of HIV infection in London's prostitutes. One report, citing police sources, suggested that $75 \%$ of one group of prostitutes in an area of London were infected with HIV. We have commented on these reports elsewhere. ${ }^{7}$ Clearly, there are prostitutes working in London who are infected with HIV, but they appear to be a small minority. The common use of condoms reported in this and other studies suggests that prostitutes are unlikely to be a major source of HIV for clients at this time. However, the high lifetime risk of gonorrhoea and the number of women with a sexually transmitted infection at the time of interview suggest that safer behaviour with clients does not eliminate the risk of sexually transmitted infections in these women.

The presence of a sexually transmitted infection was not associated with numbers of clients, duration of prostitution, or condom failures but with age and increasing numbers of non-paying partners. While these associations cannot be generalised to the group of women recruited through fieldwork who did not have a full screen for sexually transmitted infections, the two groups were similar with respect to their reported history of gonorrhoea. The prevalence of acute sexually transmitted infections (14\%) may be an overestimate since some women were recruited through attendance at the genitourinary medicine clinic, which may have been prompted by the presence of symptoms, but the associations are consistent with findings from our longitudinal study of prostitutes, in which the incidence of sexually transmitted infections is related to non-commercial sex. ${ }^{8}$

Interventions to reduce the risk of HIV and sexually transmitted infections in prostitutes generally focus on prostitutes as transmitters of disease and therefore on promoting the use of condoms for commercial sex. This is an essential message, but it needs to be complemented with the promotion of safer practices in non-commercial sex. In addition, the high numbers of women reporting condom failures suggests that promoting the use of condoms in commercial sex may not provide secure long term protection. The combination of this with the lack of use of condoms in noncommercial sex and a history of sexually transmitted infections suggests that the low prevalence of infection with HIV observed may reflect the current stage of the epidemic. It will therefore be crucial to continue preventative initiatives and further studies of the prevalence of infection in order to detect changes in the epidemic.

Paradoxically, the increasing use of condoms for commercial sex seen in the past seven years has made the promotion of condoms in non-commercial relationships more difficult. Prostitutes and their partners rarely use condoms as they are increasingly identified

\section{Public health implications}

- Prostitutes have been assumed to be at increased risk of HIV and other sexually transmitted infections, but in Britain studies have shown a low prevalence of HIV in female prostitutes

- This study confirmed a low prevalence $(0.9 \%)$ of HIV in a diverse sample of female prostitutes in London but found a higher prevalence of other sexually transmitted infections

- The women reported high levels of condom use in commercial sex but little use with noncommercial partners

- Risk of sexually transmitted infections was significantly associated with non-commercial partnerships

- Prevention campaigns and the provision of services for prostitute women should address risk reduction in general not just in commercial sex

with commercial sex. Alternative forms of protection such as spermicides or virucides may provide scope for interventions.

We thank all the participants for their help with our research; the Medical Research Council for support; the Jefferiss Research Trust for facilities; Don Jeffries and the Departments of Virology and Microbiology, St Mary's, for diagnostic services; Phillip Mortimer at the Public Health Laboratory Service for saliva testing; David Taylor-Robinson at the MRC Clinical Research Centre for chlamydia testing and Jonathan Weber and Adrian Renton for critical reading of the manuscript.

1 European Working Group on HIV Infection in Female Prostitutes. HIV infection in European female sex workers: epidemiological link with use of infection in European female sex workers: epidemich

2 McKeganey N, Barnard M, Leyland A, Coote I, Follet E. Female streetworkin prostitution and HIV infection in Glasgow. BMF 1992;305:801-4.

3 Morgan Thomas R, Plant MA, Plant MC, Sales DI. Risks of AIDS amon workers in the sex industry: some initial results from a Scottish study. $B M$ 1989;299:148-9.

4 Day S, Ward H, Harris JRW. Prostitute women and public health. $B M$ 1988;297:1585.

5 Day S, Ward H. The Praed Street Project: a cohort of prostitute women in London. In: Plant M, ed. AIDS, drugs and prostitution. London: Routledge, 1990:61-75.

6 Johnson AM, Parry JV, Best SJ, Smith AM, de Silva M, Mortimer PP. HIV surveillance by testing saliva. AIDS 1988;2:369-71.

7 Ward H, Day S. Recording HIV status on police computers. BMf 1992;304: 1635-6.

8 Ward H, Day S, Dunlop L, Donegan C, Whitaker L, de la Court A Commercial sex and HIV risk: a six year study of female sex workers. In Harvard-Amsterdam Conference, ed. Proceedings of VIII International Conference on AIDS/III STD World Congress, Amsterdam, The Netherlands 19-24 fuly 1992. Amsterdam: Congrex Holland BV, 1992:C276. (Abstract 19-24 fuly 1992.0
PoC 4186.$)$

(Accepted 26 May 1993) 